\title{
Synthesis of Poly-N-Vinylcarbazole by
}

\section{Direct and Indirect Electrolysis}

\author{
J.M. Reyna, M. Aguilar, P. Roquero* \\ Facultad de Química,Universidad Nacional Autónoma de México, \\ Ciudad Universitaria, 04510 México D.F.
}

Received 19 April 2004; accepted 21 September 2004

\begin{abstract}
Poly-N-vinylcarbazole (PNVCz) can be obtained by two different ways: when the reaction takes place through the vinyl group of the monomer, a white, linear-chain polymer, with interesting photoconducting properties, is obtained. When the polymerization occurs through the aromatic rings, the main product is a green, crosslinked polymer.

Polymerization of N-vinylcarbazole (NVC) is possible by electrochemical oxidation. Direct anodic oxidation leads mainly to the green form of the polymer. Protons released from the initial oxidation of the aromatic rings, induce the polymerization through the vinyl group. Indirect electrolysis, making use of an intermediate to achieve oxidation of the monomer, is also possible. In particular, the electrochemical generation of Ce(IV) allows to increase selectivity towards the linear-chain polymer, and can be the basis for the development of a new process, involving the anodic oxidation, in aqueous phase, of $\mathrm{Ce}(\mathrm{III})$ to $\mathrm{Ce}(\mathrm{IV})$, which will activate the polymerization reaction through the vinyl group.

This work presents results from initial characterization, by cyclic voltammetry, of the reacting system. The anodic polymerization of the monomer (NVC) was carried out in acetonitrile, using sodium perchlorate as supporting electrolyte, in the presence and in the absence of cerium salts. The voltammetric response of $\mathrm{Ce}$ (III) in aqueous phase, as a possible initiator for the polymerization, is also presented.
\end{abstract}

Keywords: poly-N-vinylcarbazole, direct and indirect electrolysis, cerium intermediate.

\footnotetext{
* Corresponding author. E-mail address: p_roquero@entropiahumana.com
} 


\section{Introducción}

Debido a su importancia en el diseño de tecnologías para la xerografía, el poli-Nvinilcarbazol de cadena lineal es el polímero foto-conductor de más intensivo uso. Las inusuales propiedades foto-electrónicas de este polímero fueron reportadas en 1957 [1]. Desde entonces, el poli(N-vinilcarbazol) ha sido citado extensivamente en la literatura técnica y de patentes. Estos estudios industriales y académicos han sido de mucha importancia para entender el fenómeno fotoelectrónico en sólidos orgánicos y poliméricos [2,3].

La preparación del poli(N-vinilcarbazol) por polimerización electroquímica se puede seguir dos vías: durante la electrólisis directa del N-vinilcarbazol, un polímero verde oscuro, entrecruzado y electroconductor, se deposita en la superficie del electrodo, mientras que, al mismo tiempo, la polimerización a través del grupo vinilo resulta en un polímero blanco, de cadena lineal, que se obtiene como precipitado [4]. La formación del polimero lineal, y una estructura propuesta para el polímero cruzado, son presentadas en la Fig. 1.

a)

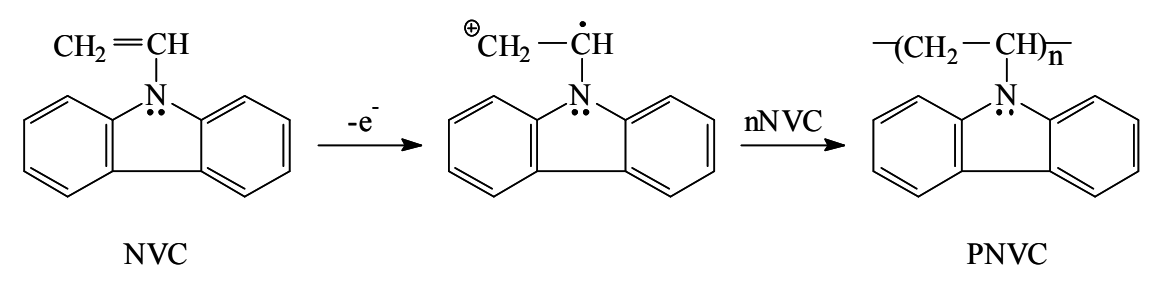

b)

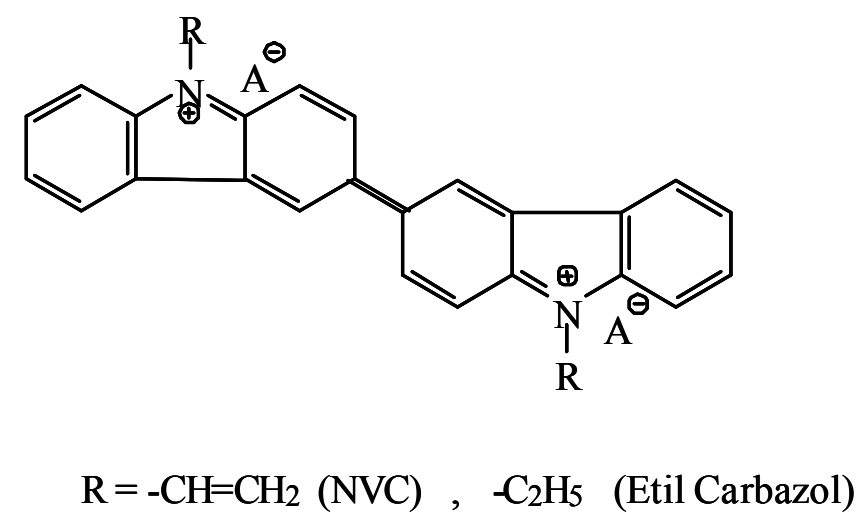

Figura 1. Polimerización del N-vinilcarbazol (NVC) a través del grupo vinilo (a). Estructura posible del producto obtenido por polimerización a través de los anillos aromáticos (b). 
El poli(N-vinilcarbazol), es un material termoplástico transparente que presenta una temperatura de transición vítrea, $\mathrm{Tg}$, de $211^{\circ} \mathrm{C}$, y se empieza a descomponer por encima de $\operatorname{los} 300^{\circ} \mathrm{C}$. Este material quebradizo polimérico exhibe muy buenas propiedades fotoconductoras, opto-electrónicas y electro-luminiscentes. Se disuelve en hidrocarburos aromáticos y clorados, y en tetrahidrofurano (THF). Es insoluble en agua, hidrocarburos alifáticos y alcoholes [5-7].

A pesar del gran interés en la preparación electroquímica de este polímero, se ha puesto muy poca atención en la polimerización oxidativa del N-vinilcarbazol empleando sales metálicas. El uso del par redox $\mathrm{Ce}(\mathrm{IV}) / \mathrm{Ce}(\mathrm{III})$ activado por electrólisis puede permitir el aumento de la selectividad hacia el polímero lineal [5]. La concentración de $\mathrm{Ce}(\mathrm{IV})$ puede mantenerse constante mediante la electrólisis. Como la oxidación electroquímica de $\mathrm{Ce}(\mathrm{III})$ a $\mathrm{Ce}(\mathrm{IV})$ se realiza simultáneamente en el ánodo, se puede prevenir la deposición del polímero verde entrecruzado en la superficie del electrodo, el cual inhibe la formación del polímero lineal. Esta forma de polimerización es una electrólisis indirecta del monómero. La Fig. 2 ilustra los esquemas de reacción para las electrólisis directa e indirecta.

En este trabajo se presenta el estudio del comportamiento electroquímico del Nvinilcarbazol, en presencia y en ausencia de sales de cerio, realizado por voltamperometría cíclica en acetonitirlo, así como los estudios iniciales del sistema reaccionante cuando se utilizan dos fases líquidas para iniciar la reacción: en la fase acuosa se oxida $\mathrm{Ce}(\mathrm{III})$ en $\mathrm{Ce}(\mathrm{IV})$, especie que inicia la polimerización en la intercara entre las dos fases líquidas.

\section{Procedimiento experimental}

Para los experimentos en fase orgánica se utilizó acetonitirlo (Aldrich), secado por destilación sobre pentóxido de fósforo, como solvente. Se utilizó $\mathrm{NaClO}_{4} 0.1$ M como electrolito soporte. La concentración de monómero se mantuvo en 0.001 M. Como iniciador se utilizó nitrato cérico de amonio (NCA, Aldrich), con una concentración de $0.001 \mathrm{M}$. El electrodo de trabajo utilizado es una hoja de 
platino, el contraelectrodo es un alambre de platino, y como referencia se utilizó un electrodo $\mathrm{Ag} / \mathrm{AgCl}$. Los voltamperogramas fueron registrados utilizando un potenciostato Voltalab 50. Cada solución fue purgada con $\mathrm{N}_{2}$, en la celda, durante 20 minutos antes del inicio de los experimentos.

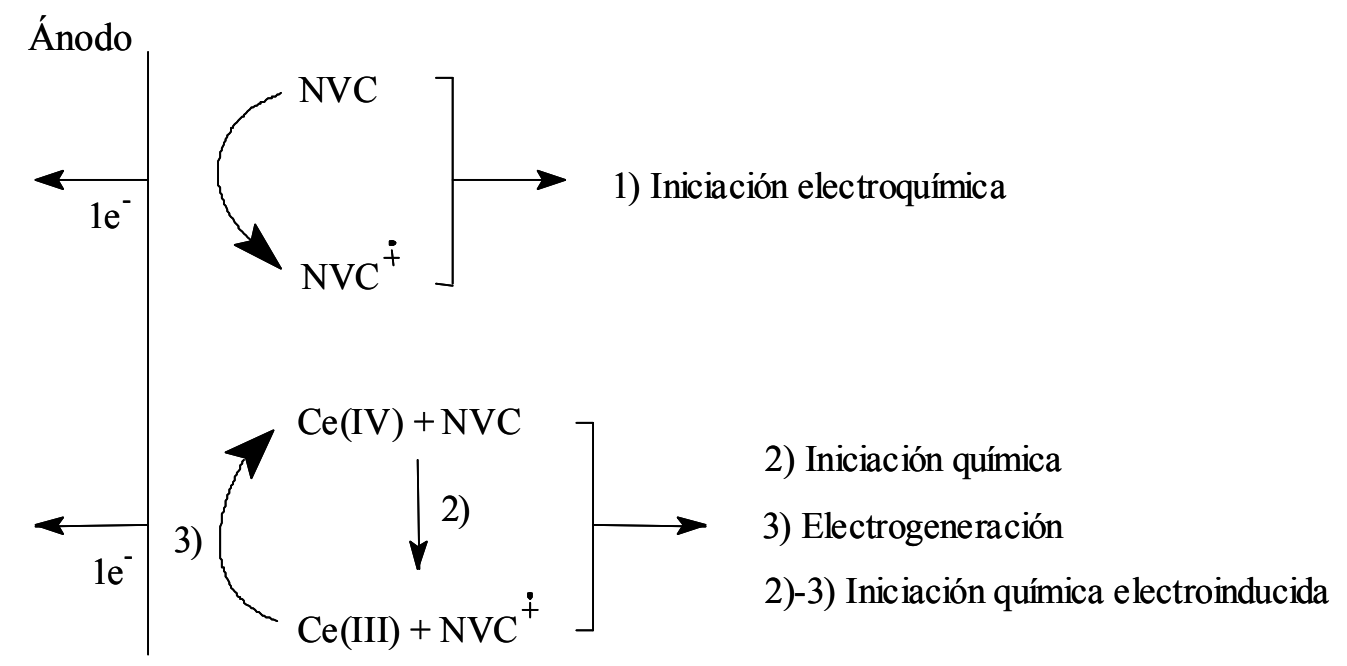

Figura 2. Esquemas reaccionantes para las electrólisis directa e indirecta del Nvinilcarbazol (NVC).

Para los experimentos con dos fases líquidas, la fase acuosa contenía $\mathrm{CeCl}_{3} 0.1$ M y $\mathrm{NaCl} 0.1 \mathrm{M}$. Una capa delgada de la fase orgánica fue colocada con una pipeta por encima de la fase acuosa. Esta fase está compuesta de acetonitirlo y monómero $0.001 \mathrm{M}$. Los electrodos sólo están en contacto con la fase acuosa. La Fig. 3 es una representación de este arreglo.

\section{Resultados y discusión}

En las Fig. 4 a 7 son presentados los resultados de voltamperometría cíclica en acetonitrilo. La Fig. 8 muestra un ejemplo de voltamperograma de la oxidación de $\mathrm{Ce}(\mathrm{III})$ en fase acuosa. En la Fig. 9 se presenta el comportamiento voltamperométrico del 9-etil carbazol, un monómero de estructura similar al NVC, pero en el que no es posible la polimerización a través de un grupo vinílico. 


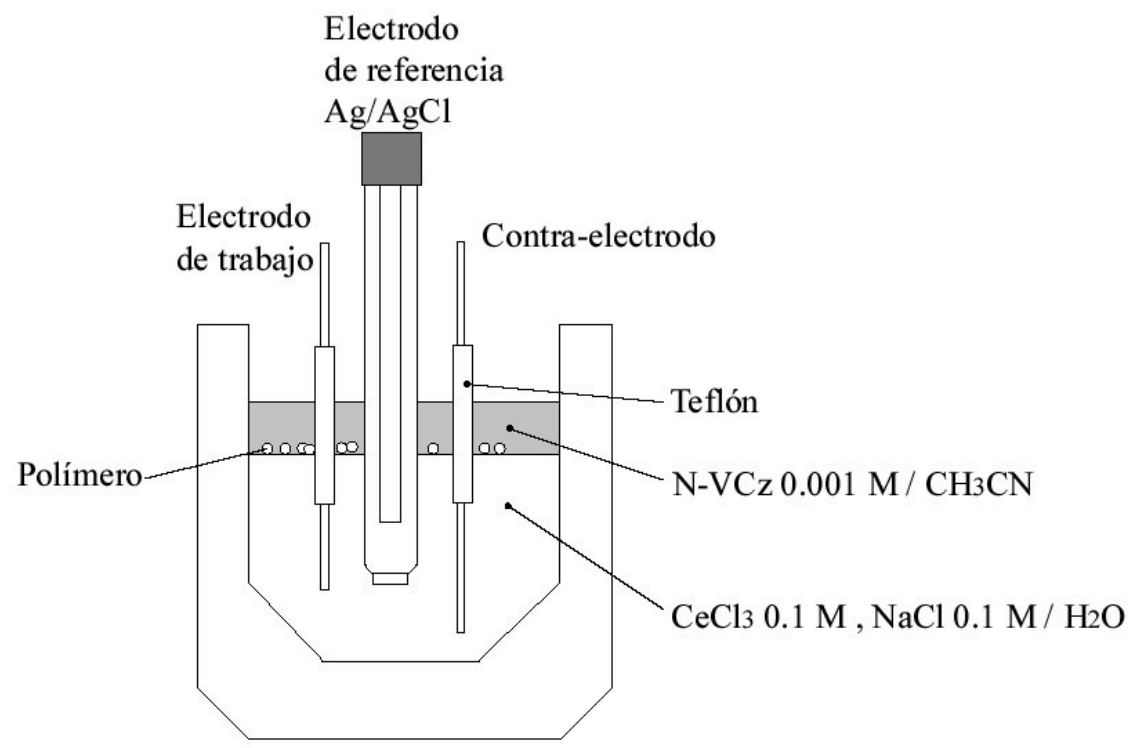

Figura 3. Celda de tres electrodos utilizada para experimentos con dos fases líquidas.

La comparación de las Fig. 4 y 5, revela 4 picos de oxidación, siendo aquel con el potencial de $1.2 \mathrm{~V}$ vs. $\mathrm{Ag} / \mathrm{AgCl}$, el que corresponde a la oxidación de $\mathrm{Ce}(\mathrm{III})$ en $\mathrm{Ce}(\mathrm{IV})$.

La Fig. 6 es una representación de la polimerización anódica directa del Nvinilcarbazol. Se puede observar que el primer ciclo presenta tres picos de oxidación en potenciales de $1.23,1.61$ y $1.76 \mathrm{~V}$ vs. $\mathrm{Ag} / \mathrm{AgCl}$. El primer pico corresponde a la oxidación inicial, del monómero, y al anclaje de la primera película de polímero sobre la superficie del electrodo.

En ciclos subsecuentes el primer pico desaparece y es remplazado por un pico más ancho, en un potencial de $1.45 \mathrm{~V}$ vs. $\mathrm{Ag} / \mathrm{AgCl}$, el segundo pico queda enmascarado, y el tercer pico se mantiene en el mismo valor de potencial $(1.76 \mathrm{~V}$ vs. $\mathrm{Ag} / \mathrm{AgCl})$.

En el segundo y tercer ciclo, la corriente eléctrica comienza a crecer en valores de potencial menores que para el primer ciclo, pues el polímero es más fácil de oxidar que el monómero. Estos resultados corresponden a la formación del polímero verde, por electrólisis direta sobre el ánodo. 


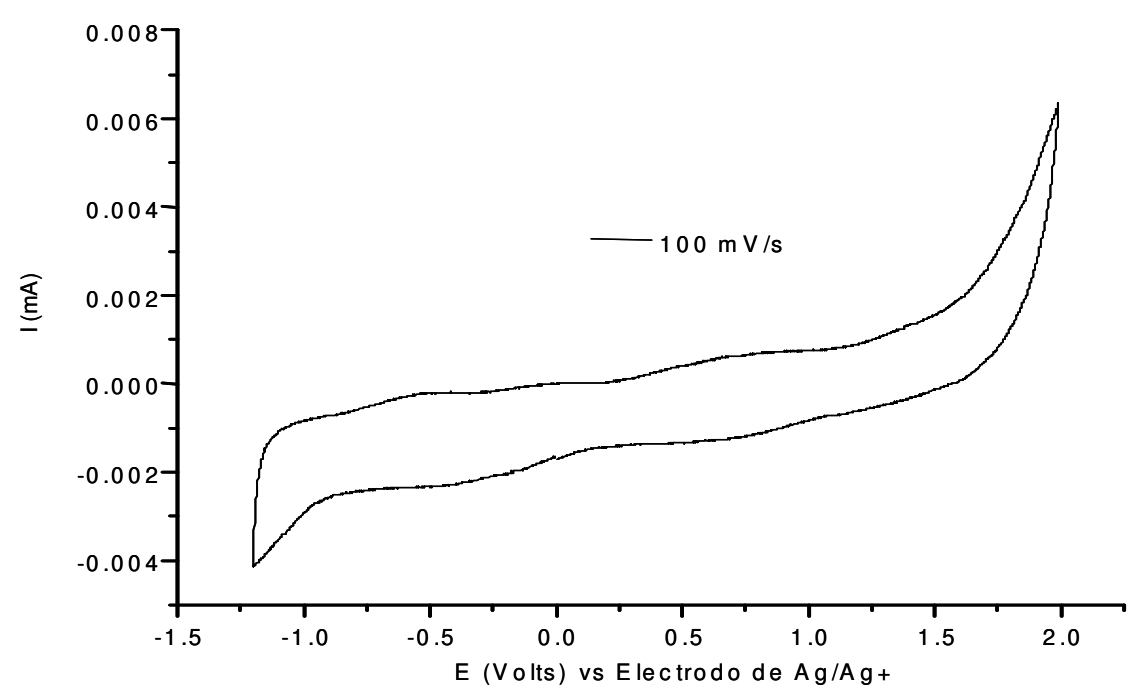

Figura 4. Voltamperograma del electrolito soporte: $\mathrm{NaClO}_{4} 0.1 \mathrm{M} / \mathrm{CH}_{3} \mathrm{CN} / \mathrm{Pt}$.

La Fig. 7 muestra el resultado de voltamperometría para una solución que contiene el monómero y el iniciador, y la reacción ocurrirá independientemente del barrido de potencial. El primer ciclo revela 3 picos anódicos en los mismos potenciales en que fueron observados para el monómero solo, pero es el pico de $1.61 \mathrm{~V}$ vs. $\mathrm{Ag} / \mathrm{AgCl}$ el que alcanza una mayor corriente eléctrica. En el segundo ciclo se observa un aumento de corriente para el primer pico y el segundo pico queda enmascarado. El tercer pico $(1.76 \mathrm{~V}$ vs. $\mathrm{Ag} / \mathrm{AgCl})$ es visible, como en el caso del monómero solo. En este caso observamos la formación de polímero por oxidación anódica directa y también la formación del polímero en solución debido a la presencia de Ce(IV).

En la Fig. 8 se presentan los resultados obtenidos para la oxidación de $\mathrm{Ce}(\mathrm{III})$ en fase acuosa. La oxidación de Ce(III) en Ce(IV) es visible en un potencial de 1.39 $\mathrm{V}$ vs. $\mathrm{Ag} / \mathrm{AgCl}$, muy cercano al potencial de oxidación del agua.

Los experimentos llevados a cabo con dos fases líquidas (Fig. 3), aplicando un potencial de $1.4 \mathrm{~V}$ vs. $\mathrm{Ag} / \mathrm{AgCl}$ al electrodo de trabajo, pusieron en evidencia la posibilidad de obtener el polímero lineal (blanco), en la fase orgánica, realizando la activación del intermediario en la fase acuosa. En este caso, no se observa la formación del polímero reticulado (verde). 


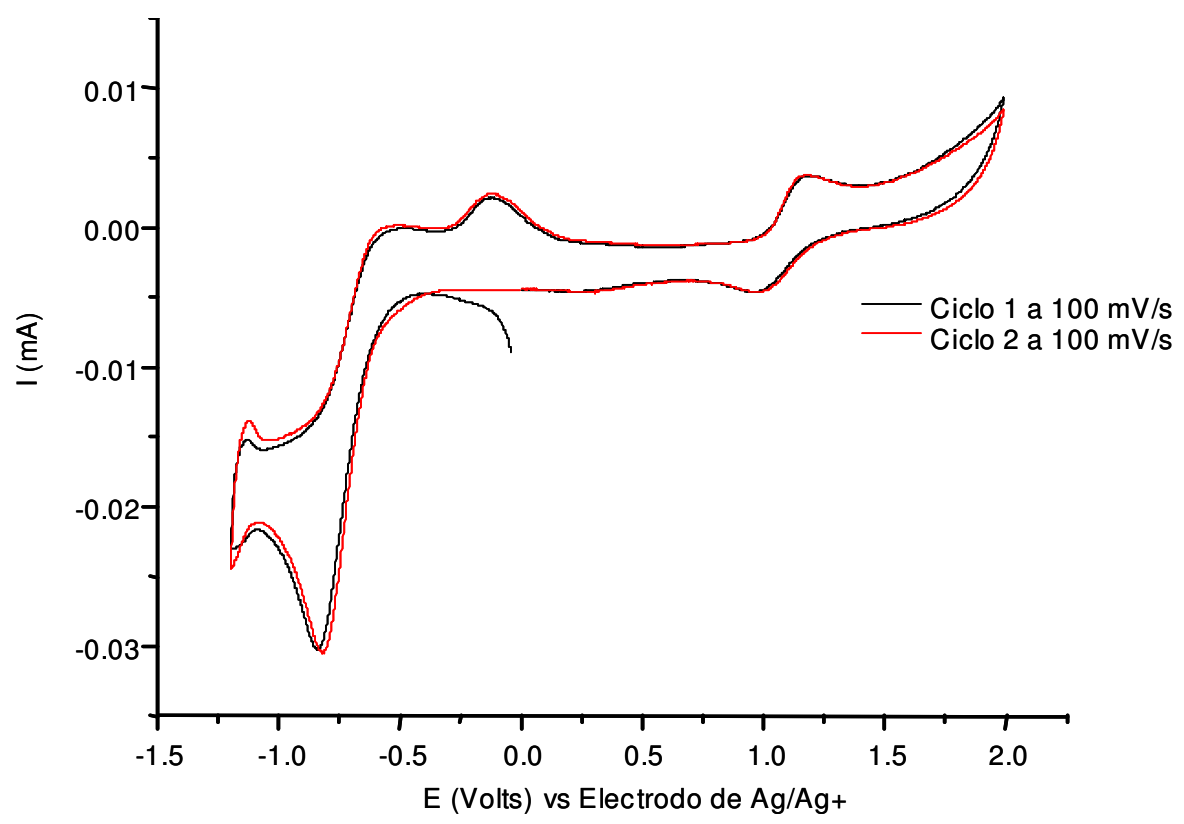

Figura 5. Voltamperograma del nitrato cérico de amonio: $\left(\mathrm{NH}_{4}\right)_{2}\left[\mathrm{Ce}\left(\mathrm{NO}_{3}\right)_{6}\right] 0.001 \mathrm{M}$, $\mathrm{NaClO}_{4} 0.1 \mathrm{M} / \mathrm{CH}_{3} \mathrm{CN} / \mathrm{Pt}$.

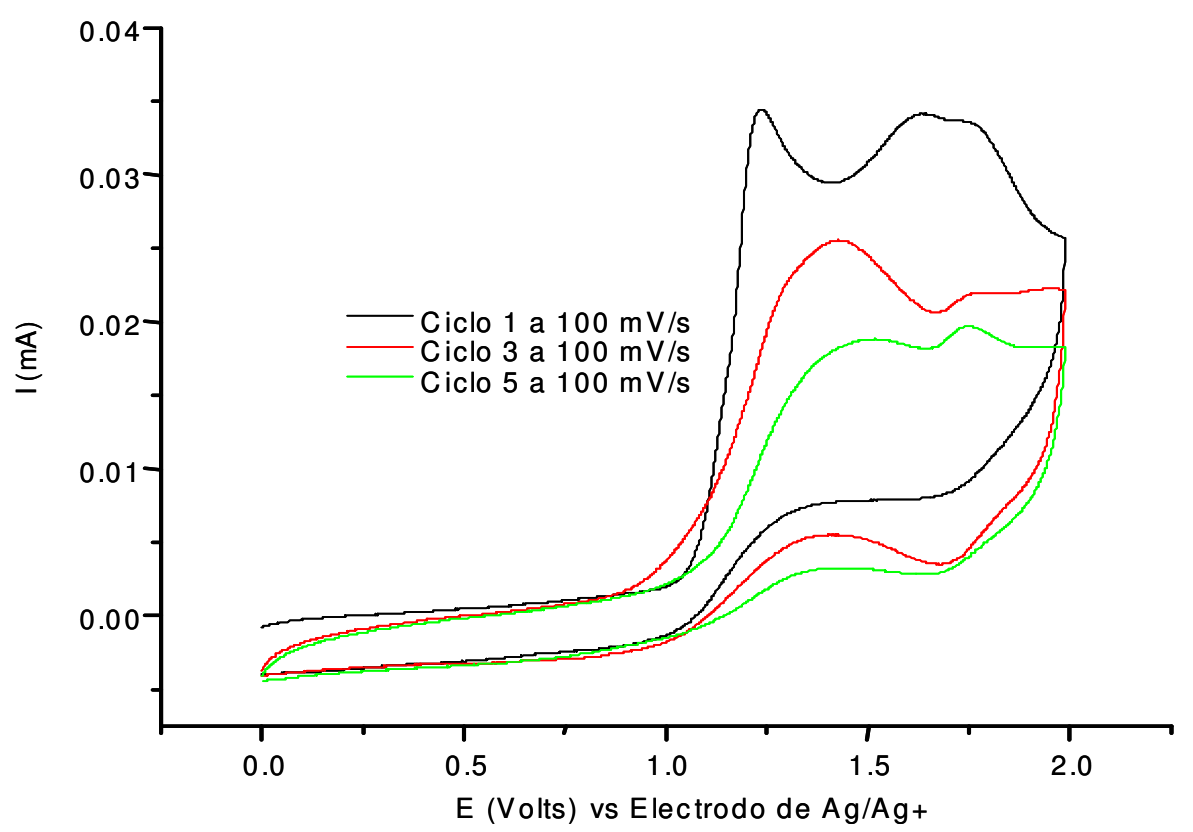

Figura 6. Voltamperograma del N-vinilcarbazol: NVC $0.001 \mathrm{M}, \mathrm{NaClO}_{4} \quad 0.1 \mathrm{M} /$ $\mathrm{CH}_{3} \mathrm{CN} / \mathrm{Pt}$. 


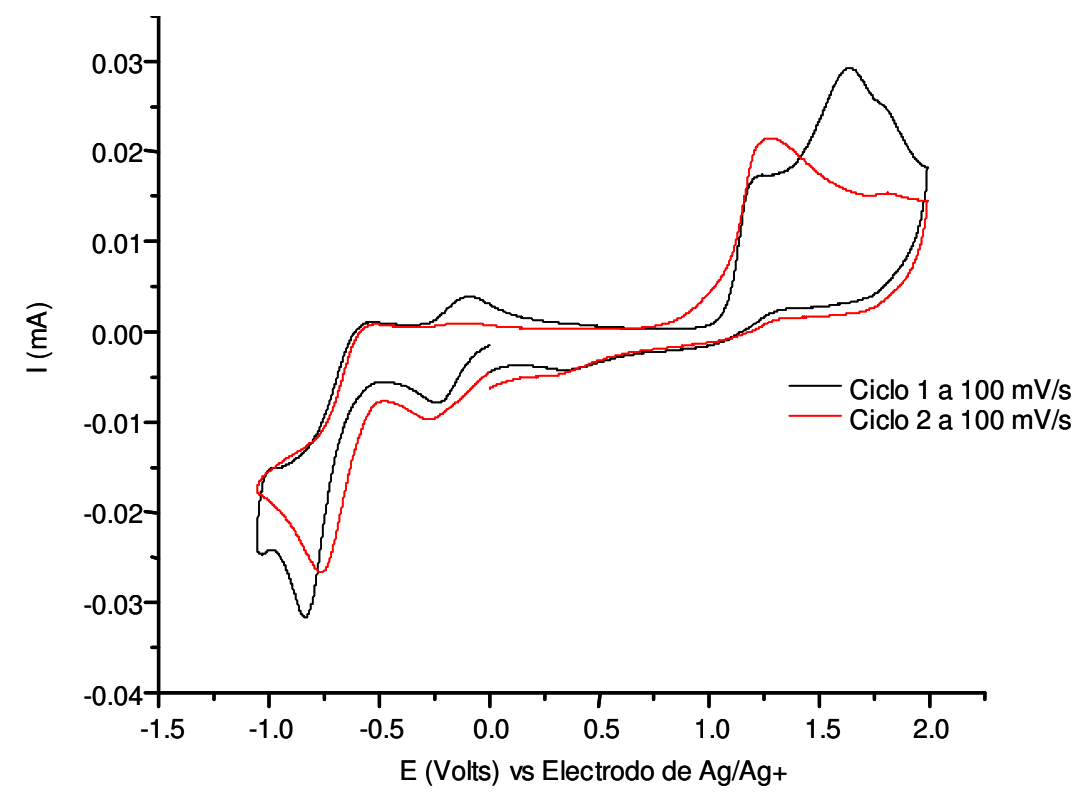

Figura 7. Voltamperograma de monómero e iniciador: $\left(\mathrm{NH}_{4}\right)_{2}\left[\mathrm{Ce}\left(\mathrm{NO}_{3}\right)_{6}\right] 0.001 \mathrm{M}$, NVC $0.001 \mathrm{M}, \mathrm{NaClO}_{4} 0.1 \mathrm{M} / \mathrm{CH}_{3} \mathrm{CN} / \mathrm{Pt}$.

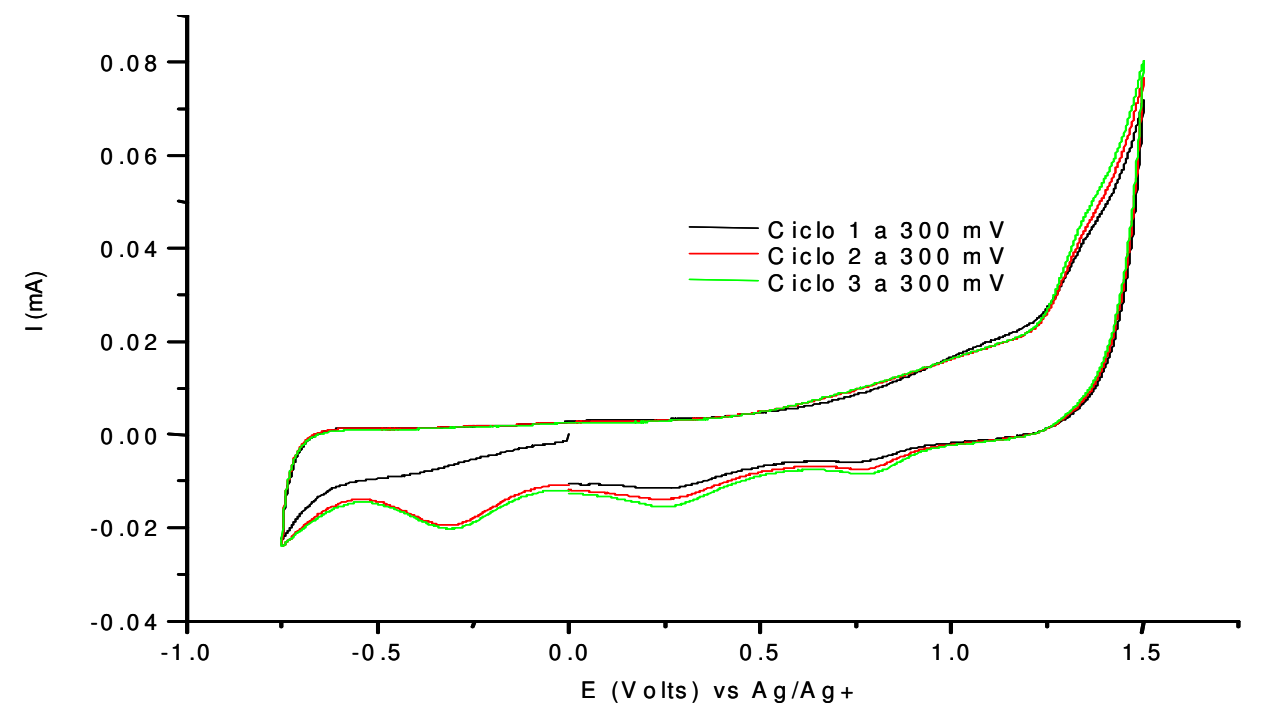

Figura 8. Voltamperograma de cloruro de cerio en fase acuosa: $\mathrm{CeCl}_{3} 0.1 \mathrm{M}, \mathrm{NaCl} 0.1$ $\mathrm{M} / \mathrm{H}_{2} \mathrm{O} / \mathrm{Pt}$. 


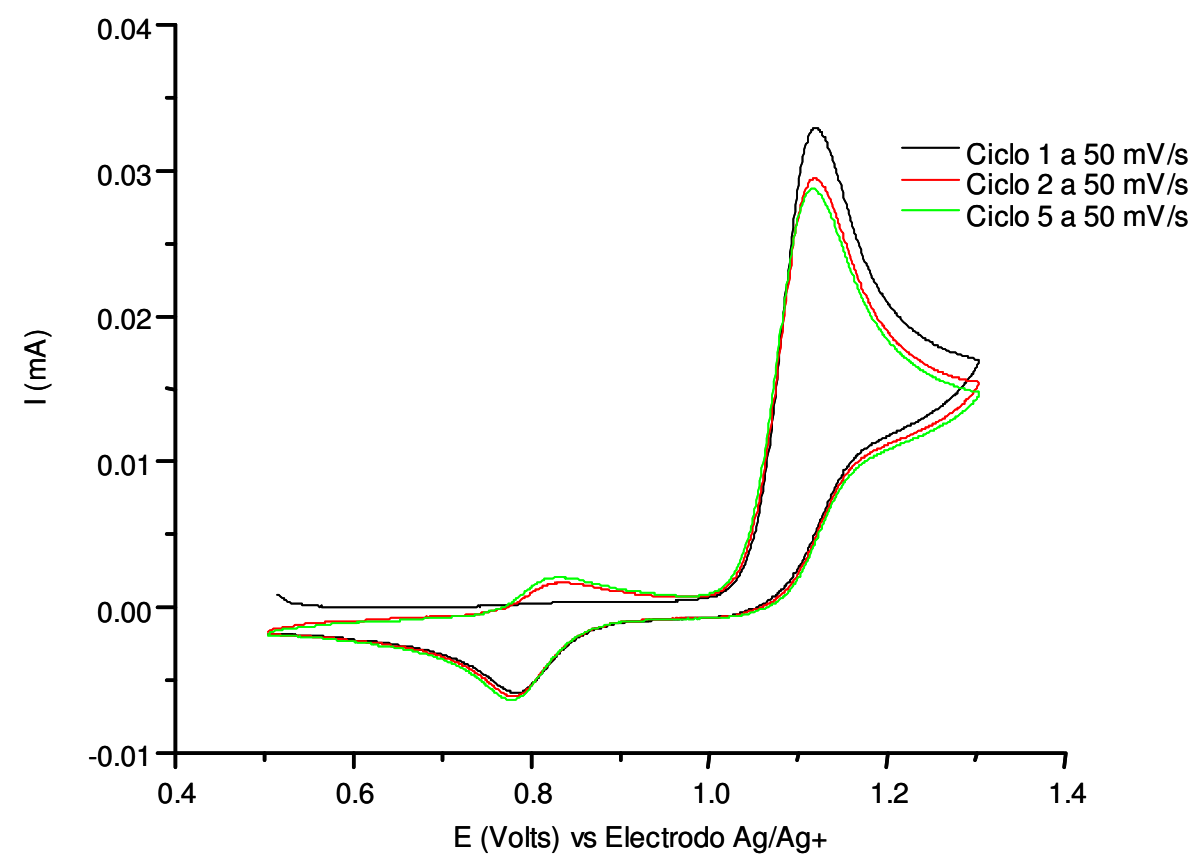

Figura 9. Voltamperograma del N-etilcarbazol: $\mathrm{NEC} 0.001 \mathrm{M}, \mathrm{NaClO}_{4} 0.1 \mathrm{M} / \mathrm{CH}_{3} \mathrm{CN}$ / Pt.

\section{Conclusión}

El poli-N-vinilcarbazol de cadena lineal es un polímero fotoconductor importante en procesos xerográficos. La electrólisis indirecta es un método adecuado para obtener este polímero con alta selectividad. El cerio puede ser utilizado para la iniciación de esta reacción. Puesto que Ce(III) es soluble en fase acuosa, y $\mathrm{Ce}(\mathrm{IV})$ lo es en solventes orgánicos, la combinación de dos fases líquidas permitiría el diseño de un proceso electroquímico para la síntesis de este polímero, pero se requiere lograr una oxidación eficiente del $\mathrm{Ce}$ (III) en fase acuosa, para lo cual será necesario investigar esta reacción sobre ánodos con alta sobretensión de oxígeno. 


\section{Síntesis de Poli-N-Vinilcarbazol por Electrólisis Directa e Indirecta}

\section{Resumen}

El poli-N-vinilcarbazol (PNVC) puede obtenerse por dos vías diferentes: cuando la polimerización se lleva a cabo por el grupo vinilo del monómero, se obtiene un polímero lineal, de color blanco, con interesantes propiedades como fotoconductor. Cuando la polimerización ocurre a través de los anillos aromáticos, se obtiene un polímero reticulado, de color verde, electroconductor. El polímero blanco es utilizado en importantes aplicaciones en equipos xerográficos.

La polimerización del N-vinilcarbazol (NVC) puede ser iniciada por oxidación electroquímica. Cuando el monómero sufre una oxidación anódica directa, la polimerización ocurre inicialmente por medio de los anillos aromáticos, obteniendo el polímero verde. Los protones liberados en esta etapa reaccionan con los grupos vinilo, iniciando la polimerización de estos por un mecanismo de tipo radical-catiónico. Por otra parte, se ha observado que el uso de un iniciador en solución, por ejemplo una sal de $\mathrm{Ce}(\mathrm{IV})$, permite aumentar la selectividad de la reacción hacia el polímero lineal. De esta manera es posible diseñar un proceso para la síntesis del polímero más interesante, el de cadena lineal, mediante la selección de un proceso por electrólisis indirecta, en que se genere anódicamente Ce(IV), el cual iniciará la polimerización por los grupos vinilo. En este trabajo se presentan los resultados de la caracterización inicial, por voltamperometría cíclica, del sistema reaccionante. Se estudia la polimerización anódica del monómero en acetonitirilo, utilizando perclorato de sodio como electrolito soporte, en ausencia y en presencia de sales de cerio. Se presenta también el estudio de la electrólisis de $\mathrm{Ce}(\mathrm{III})$ en fase acuosa como posible iniciador de la reacción.

Palabras clave: poli-N-vinilcarbazol, electrólisis directa e indirecta, intermediario de cerio.

\section{Referencias}

1. M. Aldissi, Inherently Conducting Polymers, Noyes Data Corporation, New Jersey, USA, 1989.

2. V. Papež, J. Electroanal. Chem. 282(1) (1990) 23.

3. V. Papež, M. Josowicz, J. Electroanal. Chem. 365 (1994) 139.

4. A.S. Saraç, E. Sezer, B. Ustamehmetoğlu, Polym. Adv. Technol. 8 (1997) 556. 
5. R.G. Linford, Electrochemical Science and Technology of Polymers, Elsevier Applied Science, New York, USA, 1990.

6. R. Liepins, C. Chen, Electrical Properties of Polymers, Chemical Principles, Hanser Publishers, Munich, Alemania, 1987.

7. S. Vico, V. Carlier, C. Buess-Herman, J. Electroanal. Chem. 1 (1999) 475. 